\title{
DOENÇA ONCOLÓGICA: PERFIL EPIDEMIOLÓGICO EM UNIDADE DE REFERENCIA NA AMAZÔNIA
}

Walter de Souza Tavares ${ }^{1}$

Lucyelle Gleyce Ferreira Pádua ${ }^{1}$

Mayrla Santana Picanço ${ }^{1}$

Francineide Pereira da Silva Pena ${ }^{1}$

Maria Virginia Filgueiras de Assis Melo' https://orcid.org/0000-0002-8268-6207

https://orcid.org/0000-0001-6980-0848

https://orcid.org/0000-0001-9540-6289

https://orcid.org/0000-0001-8465-4252

https://orcid.org/0000-0002-5260-942X

Objetivo: Identificar o perfil epidemiológico, sociodemográfico, clínico e os cuidados de enfermagem no seguimento de pessoas com doença oncológica no estado do Amapá. Metodologia: Estudo transversal, descritivo, documental. Os dados foram coletados dos prontuários, por roteiro sistematizado, na amostra de 320 prontuários, no período de 2014 a 2018. Resultados: Predomínio do sexo feminino (55\%), faixa etária entre 60 - 69 anos (21\%), escolaridade ensino fundamental incompleto (40\%). O câncer de colo uterino (33\%) foi predominante no sexo feminino, e o de próstata (30\%) no sexo masculino. Conclusão: O perfil identificado no Amapá para a população masculina foi o câncer de próstata, que se semelhou ao perfil nacional, já para a população feminina, fugiu do perfil nacional, onde o câncer de mama responde pelo topo do ranking, enquanto no Amapá é o de colo uterino.

Descritores: Neoplasias; Epidemiologia; Enfermagem.

\section{ONCOLOGICAL DISEASE: EPIDEMIOLOGICAL PROFILE IN A REFERENCE UNIT IN THE AMAZON}

Objective: To identify the epidemiological, sociodemographic, clinical profile and the nursing care in the follow up of peoples with oncological disease in the state of Amapá. Methodology: cross-sectional, descriptive, documentary study. The data were collected from the medical records by a systematized script, with a sample of 320 records of interventions that occurred in the period from 2014 to 2018. Results: The predominance was female (55\%), aged over $60(21 \%)$, incomplete primary schooling (40\%). Cervical cancer was the most identified (33\%) in the female sex, and the prostate cancer (30\%) in the male sex. Conclusion: The profile identified in Amapá for the male population admits prostate cancer, a characteristic similar to the national profile, already for the female population, runs away from the national profile, where breast cancer is at the top of the ranking, while in Amapá it is the uterine cervix.

Descriptors: Neoplasms; Epidemiology; Nursing.

\section{ENFERMEDAD ONCOLÓGICA: PERFIL EPIDEMIOLÓGICO EN UNA UNIDAD DE REFERENCIA EN LA AMAZONİA}

Objetivo: Identificar el perfil epidemiológico, sociodemográfico, clínico y la atención de enfermería en el seguimiento de personas con enfermedad oncológica en el estado de Amapá.Metodología: Estudio transversal, descriptivo, documental. Los datos fueron recolectados de los prontuarios, por itinerario sistematizado, teniendo una muestra de 320 prontuarios, de intervenciones ocurridas en el periodo de 2014 a 2018. Resultados: Ocurrió el predominio del sexo femenino (55\%), del grupo de edad superior a los 60 (21\%) de la escolaridad escolar incompleta (40\%). El cáncer de cuello uterino fue el más identificado (33\%) en el sexo femenino, y el de próstata (30\%) en el sexo masculino. Conclusión: El perfil identificado en Amapá para la población masculina admite el cáncer de próstata, característica similar al perfil nacional, ya para la población femenina, huye del perfil nacional, donde el cáncer de mama es el que está en la cima del ranking, en el Amapá es el de cuello uterino.

Descriptores: Neoplasias; Epidemiología; Enfermería.

IUniversidade Federal do Amapá,AP, Brasil.

Autor Correspondente: Walter de Souza Tavares Email:Walter_sou_z@hotmail.com Recebido: 01/3/20 Aceito: 12/6/20 


\section{INTRODUÇÃO}

Para a Organização Mundial de Saúde, o câncer é considerado problema de saúde pública, decorrente de causas múltiplas, como os fatores ambientais, culturais, socioeconômicos e estilo de vida, e anualmente responde por 8,8 milhões de óbitos, sendo a maioria em países de baixa e média renda ${ }^{1}$

Conforme o Instituto Nacional do Câncer (INCA), a prevenção e controle do câncer representam desafio amplo e complexo a julgar pela diversidade regional e grandeza continental do Brasil. A estimativa é de que ocorra aumento na incidência, mortalidade e morbidade da população com doença oncológica no biênio 2018-2019 quando comparado a período anterior, indicando importante avaliação do perfil epidemiológico destes para a compreensão de futuros investimentos em pesquisas e para ações efetivas de prevenção².

Os cânceres que mais afetam a população masculina do Brasil, são os de próstata, traqueia, brônquio e pulmão, cólon e reto e os que mais atingem a feminina, são os cânceres de mama, cólon e reto, colo de útero ${ }^{3}$. No Amapá, as estimativas se apresentam próximas à realidade da população brasileira, no entanto, o cenário obtém certas excepcionalidades, como maior frequência de câncer de colo de útero.

Diante da expressividade do tema, é imprescindivel a procura por dados de qualidade e informações reais, além do mais, a compreensão dos parâmetros envolvidos no cotidiano de pessoas com doença oncológica, tais quais: as ações de enfermagem no tratamento, o perfil epidemiológico e a demanda sociodemográfica no estado do Amapá remetem necessidade de maiores discussões sobre o assunto. Diante disso, trabalhar aspectos epidemiológicos auxiliam na distribuição da ocorrência da doença oncológica e nas ações de controle, visando a promoção de saúde, bem como na verificação da eficácia das políticas públicas existentes ou na criação futura das mesmas por órgãos responsáveis.

Neste contexto, este estudo teve como objetivo identificar o perfil epidemiológico, sociodemográfico, clínico e os cuidados de enfermagem no seguimento de pessoas com doença oncológica no estado do Amapá.

\section{METODOLOGIA}

\section{Tipo de estudo}

Trata-se de um estudo transversal com abordagem quantitativa, descritiva e documental.

\section{Local e periodo do estudo}

Foi realizado na Unidade de Alta Complexidade em On- cologia (UNACON) do Hospital de Clínicas Dr. Alberto Lima, no período de janeiro de 2014 a outubro de 2018, sendo a coleta de dados realizada no mês de novembro de 2018 .

\section{População, Amostra e critérios de elegibilidade}

A população foi composta por 3.028 prontuários ativos no arquivo da UNACON, tendo amostragem aleatória estratificada de 320 prontuários que obedeceram aos critérios de inclusão: prontuários de pessoas maiores de 18 anos, com diagnóstico de neoplasia maligna confirmada por biópsia, em seguimento entre janeiro de 2014 a outubro de 2018, que já finalizaram o tratamento ou ainda em processo (pessoas que passam por terapias de controle ao câncer, no aguardo de remissão da doença). Foram excluídos prontuários com preenchimento inadequado, com escrita ilegível e com diagnósticos recentes que não foram comprovados por biopsia.

\section{Coleta de dados}

A coleta de dados foi realizada após a autorização da direção do hospital, aprovação do Comitê de Ética em Pesquisa da Universidade Federal do Amapá (Parecer no 3.005.900) e anuência do participante, através da assinatura do Termo de Consentimento Livre e Esclarecido (TCLE). Foi aplicado um roteiro sistematizado para coleta das informações relacionadas ao sexo, idade no período, naturalidade, local de procedência, estado civil, escolaridade e profissão, dados clínicos como a hipótese diagnóstica, ano de realização da biópsia, recidiva, situação e tipo de tratamento realizado, tempo do diagnóstico até o começo do tratamento (calculado pela data da biópsia até o início do tratamento), além dos registros de enfermagem quanto à assistência prestada.

\section{Análise de dados}

Os dados foram tabulados, interpretados, processados e analisados por meio da estatística descritiva de frequência relativa e absoluta. As variáveis estado civil e escolaridade foram agrupadas por códigos, a fim de facilitar a compreensão: $\mathrm{EC}^{1}$ (não casados), $\mathrm{EC}^{2}$ (casados e/ou união estável) e $\mathrm{S}^{1}$ (sem informações); $A$ (analfabetos), $\mathrm{E}^{1}$ (pessoas que cursaram até o ensino fundamental) e $E^{2}$ (acima do ensino fundamental). Os resultados foram demonstrados em gráficos e tabelas, sendo utilizado o software estatístico Bioestat 5.4, tabulado no Microsoft Excel for Windows 2016® e discutidos à luz da literatura pertinente.

\section{RESULTADOS}

Do perfil identificado, predominou o sexo feminino 55\% (177). A faixa etária de 60 a 69 anos. Na Tabela 1 estão apresentadas as demais variáveis sociodemográficas das pessoas em seguimento. 
Tabela 1 - Identificação das pessoas com doença oncológica em seguimento na unidade de alta complexidade em oncologia segundo características sociodemográficas. Macapá/AP, 2018. N=320.

\begin{tabular}{|c|c|c|c|}
\hline \multicolumn{2}{|c|}{ Variáveis } & \multirow{2}{*}{$\begin{array}{c}\mathbf{N} \\
177\end{array}$} & \multirow{2}{*}{\begin{tabular}{|l|}
$\% *$ \\
$55 \%$ \\
\end{tabular}} \\
\hline & Feminino & & \\
\hline & Masculino & 143 & $45 \%$ \\
\hline \multirow{7}{*}{ Faixa Etária } & $<20$ a 29 & 18 & $6 \%$ \\
\hline & 30 a 39 & 33 & $10 \%$ \\
\hline & 40 a 49 & 63 & $20 \%$ \\
\hline & 50 a 59 & 61 & $19 \%$ \\
\hline & 60 a 69 & 68 & $21 \%$ \\
\hline & 70 a 79 & 53 & $16 \%$ \\
\hline & 80 a 89 & 24 & $8 \%$ \\
\hline \multirow{3}{*}{ Estado Civil } & $\mathrm{EC}^{1}$ & 106 & $33 \%$ \\
\hline & $\mathrm{EC}^{2}$ & 178 & $56 \%$ \\
\hline & $\mathrm{S}^{1}$ & 36 & $11 \%$ \\
\hline \multirow{4}{*}{ Escolaridade } & A & 41 & $13 \%$ \\
\hline & $E^{1}$ & 129 & $40 \%$ \\
\hline & $E^{2}$ & 100 & $31 \%$ \\
\hline & $\mathrm{S}^{1}$ & 50 & $16 \%$ \\
\hline \multirow{4}{*}{ Trabalho } & Sem ocupação fixa & 186 & $58 \%$ \\
\hline & Fora de casa & 76 & $24 \%$ \\
\hline & Do lar & 33 & $10 \%$ \\
\hline & Aposentados & 25 & $8 \%$ \\
\hline \multirow{7}{*}{ Procedência } & Macapá/AP & 92 & $29 \%$ \\
\hline & Afuá/PA & 37 & $12 \%$ \\
\hline & Breves/PA & 36 & $11 \%$ \\
\hline & Chaves/PA & 16 & $5 \%$ \\
\hline & Santana/AP & 11 & $3 \%$ \\
\hline & Amapá/AP & 10 & $3 \%$ \\
\hline & Outros & 118 & $37 \%$ \\
\hline
\end{tabular}

Fonte: Protocolo de pesquisa (2018) *: Percentual arredondado para múltiplos de 10. EC (não casados), EC² (casados e/ ou união estável) e $S^{1}$ (sem informações); $A$ (analfabetos), $E^{1}$ (pessoas que cursaram até o ensino fundamental) e $E^{2}$ (acima do ensino fundamental). 
A tabela 2 revela variáveis clínicas e epidemiológicas dos cinco cânceres predominantes para o sexo masculino e para o sexo feminino, identificados nos 320 prontuários que constituiu a amostra deste estudo e que tiveram diagnóstico entre os anos de 2014 a 2018.

Tabela 2 - Identificação dos tipos de cânceres predominantes segundo localização primária e sexo. Macapá/AP, 2018. N=320.

\begin{tabular}{|c|c|c|c|}
\hline \multicolumn{2}{|c|}{ Masculino } & Feminino \\
\hline Localização Primária & $\% *$ & Cocalização Primária do Útero & Mama \\
\hline Próstata & $30 \%$ & Estômago & $29 \%$ \\
\hline Estômago & $18 \%$ & Cólon e Reto & $8 \%$ \\
\hline Cólon e Reto & $8 \%$ & Ovários & $5 \%$ \\
\hline Cavidade Oral & $6 \%$ & $5 \%$ & $4 \%$ \\
\hline Traqueia, Brônquio e Pulmão & $5 \%$ & Co \\
\hline
\end{tabular}

Fonte: Protocolo de pesquisa (2018) *: Percentual arredondado para múltiplos de 10.

A maior parte das biópsias ocorreu no ano de 2017, sendo o ano com maior número de diagnósticos 37,5\% (120) das pessoas. Os casos de recidivas foram identificados 5,3\% (17) das pessoas e identificou a mesma doença ou de um novo tipo.

Os tipos de tratamentos disponíveis na unidade foram ci- rurgia e quimioterapia, entretanto, 36\% (115) da amostragem tinham variadas formas terapêuticas, como: radioterapia, quimioterapia associado à radioterapia, imunoterapia, dentre outras. Para melhor compreensão a tabela 3 apresenta as variáveis clínicas subdividas em categorias.

\begin{tabular}{|c|c|c|c|}
\hline \multirow{3}{*}{ Situação } & Seguimento & 239 & $75 \%$ \\
\hline & Paliativo & 52 & $16 \%$ \\
\hline & Não realizou & 29 & $9 \%$ \\
\hline \multirow{2}{*}{ Tipo de tratamento } & Quimioterapia & 83 & $26 \%$ \\
\hline & Cirurgia + Quimioterapia & 65 & $20 \%$ \\
\hline \multirow[t]{2}{*}{ Diagnóstico ao Tratamento } & $>1$ ano & 18 & $6 \%$ \\
\hline & Não realizou & 29 & $9 \%$ \\
\hline
\end{tabular}


$\mathrm{Na}$ tabela 4, estão identificados os cuidados de enfermagem. Observou-se pelos registros que algumas ações foram realizadas com maior frequência e os cuidados foram realizados com menor frequência.

Tabela 4 - Distribuição dos cuidados de enfermagem a pessoas em seguimento na unidade de alta complexidade oncológica. Macapá/AP, 2018. N=320

\begin{tabular}{|c|c|c|}
\hline Cuidados & N & \%* \\
\hline Monitorização e controles & 328 & $71 \%$ \\
\hline Tarefas administrativas e gerenciais & 320 & $100 \%$ \\
\hline Passagem de sondas & 26 & $8 \%$ \\
\hline Medidas de Higiene & 26 & $8 \%$ \\
\hline Cuidados com drenos & 12 & $4 \%$ \\
\hline
\end{tabular}

Fonte: Protocolo de pesquisa (2018) *: Percentual arredondado para múltiplos de 10.

\section{DISCUSSÃO}

No presente estudo destaca-se a predominância do sexo feminino entre doenças oncológicas com (55\%) dos casos. As mulheres tendem a relatar as morbidades que apresentam com maior facilidade do que os homens, assim frequentando mais aos serviços de saúde, com consequente diagnóstico e tratamento oportuno ${ }^{4}$. 0 perfil identificado de atendimento em saúde a literatura corrobora com o processo de transição demografica que ocorre globalmente, e o Brasil segue as tendências, como o aumento quantitativo de idosos e o fenômeno de feminilização do envelhecimento, devido a maior expectativa de vida das mulheres em relação aos homens ${ }^{5}$.

Relacionado a idade, predominou a população idosa, com maior frequência na faixa etária acima de 60 anos (45\%). De acordo com Carvalho et al. ${ }^{6}$, em que mais de $50 \%$ de todos os tipos de cânceres ocorrem em pessoas com mais de 65 anos, sendo a principal causa de morte em homens e mulheres com idades entre 60 a 79 anos. Entende-se que o avanço da idade produz redução da reserva funcional, da capacidade do organismo se adaptar e manter um equilíbrio energético, comprometendo os mecanismos necessarios para a realização das atividades e aumento de prevalëncia de doenças ${ }^{5}$, associado ao maior período de exposição aos diferentes agentes causadores/ agravadores da doença oncológica, aumentando sua fragilidade e tornando a doença mais incidente nesse grupo.
Sobre o estado civil, a maior proporção foi entre os casados ou em união estável, em (56\%) da amostra. A familia tem impacto significativo sobre a saude de seus membros, influenciando sobre as enfermidades que os acome$t^{7}$. Os casados ou em união estável realizam seguimento de maneira mais eficaz, devido ao apoio social recebido, sugerindo coesão familiar que contribui para desenvolver recursos e competência sociais que são características que quando vividas promovem resiliência e enfrentamento da doença ${ }^{8}$. Neste contexto, surgiram mudanças nas relações conjugais, ou seja, uma relação de maior compreensão e apoio mútuo, pois o cuidar que era direcionado a família foi substituido pelas tarefas que a doença exige, esse achado corrobora com a característica de coesão familiar, que evidencia a qualidade das relações familiares em forma de comunhão de valores, favorecendo visão de mundo para o futuro?

A população desse estudo, apresentou baixo grau de escolaridade, (13\%) eram analfabetos e (40\%) estudaram até o ensino fundamental. Essa característica identifica uma barreira para o acesso a informações de boa qualidade, ainda mais quando associada a doenças crônicas não transmissiveis nos segmentos socialmente desfavorecidos $^{10}$. Para a população feminina, é fator indicativo importante ao acesso a exames como a mamografia e citologia oncótica, nos quais a procura aumenta de acordo com o grau de escolaridade ${ }^{11}$. 
Parcela significativa (29\%) da amostra estudada revelou naturalidade da capital, Macapá. No entanto, os demais locais eram de municipios próximos a capital, incluindo alguns municípios do estado do Pará, mais especificamente a ilha marajoara do Afuá com (12\%). Quando esse deslocamento ocorre, torna-se imprescindivel refletir a dinâmica do atendimento, na perspectiva de pensar estratégias que atendam as diferentes necessidades da população.

Acerca do perfil epidemiológico, para o INCA, os três tipos de cânceres prevalentes na população masculina brasileira foram: câncer de próstata (31,7\%); câncer de traqueia, brônquio e pulmão (8,7\%); e câncer de cólon e reto $(8,1 \%)^{2}$. Já na população feminina foram: câncer de mama feminina (29,5\%); câncer de cólon e reto $(9,4 \%)$; e câncer de colo de útero $(8,1 \%)^{2}$.

No estudo em questão, os três tipos de cânceres de maior proporção na população masculina foram: câncer de próstata (30\%); câncer de estomago (18\%); e câncer de cólon e reto (8\%). Enquanto que na população feminina foram: câncer de colo do útero (33\%); câncer de mama (29\%); e câncer de estômago (8\%).

Esse perfil evidencia que os resultados deste estudo quando comparados aos dados das estimativas nacionais indicam semelhanças aos cânceres que acometem a população masculina, entretanto, se diferenciam dos tipos de canceres na população femininina.

Destacando as singularidades, o câncer de colo de útero na maioria dos casos (70\%) ocorre em áreas com menores índices de desenvolvimento humano, apresentando alta taxa de óbitos naqueles países menos desenvolvidos $^{12}$. Sendo o mais incidente na Região Norte e o terceiro a índices nacionais ${ }^{1-2}$

A variação regional e suas distintas características econômico-culturais, regionais e mesmo interurbanas encontradas no Brasil, geraram um padrão em que coexistem fatores relacionados à pobreza e ao desenvolvimento ${ }^{13}$. Com isso, a baixa condição socioeconômica interfere no acesso aos serviços de prevenção, rastreamento regular, diagnóstico e tratamentos oportunos. Existe disparidades entre as áreas que têm melhores estruturas urbanas ( $R e-$ giões Sudeste e Sul) com a presença de sistemas de saúde bem equipados e com funcionamento mais eficiente em contraponto a aquelas que possuem ausência de níveis hierárquicos intermediários (Regiões Norte e Nordeste)².

Em suma, as ações de prevenção não contemplam de maneira igualitária toda a população. Isso ocorre devido às variações regionais com suas respectivas assistências de saúde, aspecto importante no que concerne ao cân- cer de colo de útero, pois este sofre influência de fatores sociais e econômicos uma vez que as regiões menos desenvolvidas socioeconomicamente apresentam maior incidência da doença.

Neste estudo, o câncer de estômago apresentou expressividade entre as pessoas. Na população masculina, ocupou a segunda posição entre os predominantes, apontando particularidade quando analisado o cenário nacional e elevado índice de casos na população feminina estando como o terceiro predominante. O INCA, constata que é o segundo câncer mais frequente entre os homens nas regiões Norte e Nordeste, enquanto nas regiões Sul e Centro-Oeste é o quarto mais frequente e na região Sudeste, ocupa a quinta posição. Para as mulheres, é o quinto mais frequente na Região Norte e o sétimo na Região Sudeste ${ }^{2}$.

$\mathrm{Na}$ Região Norte, destacou-se as situações de risco que influenciam o surgimento do câncer de estômago: os baixos índices no tratamento de esgoto, a prevalência de infecção pela bactéria $H$. Pylori, o consumo de carnes na forma de embutidos, da farinha de mandioca e de bebidas alcoólicas $^{14}$. Assegurada a associação entre doenças oncológicas no estômago e o baixo nível socioeconômico, fator observado nas regiões Norte e Nordeste, que possuem maior incidência da doença, enfatizando que a Região Norte possui o menor índice no tratamento de água fornecido a população ${ }^{15}$

O câncer de próstata é notadamente reconhecido como problema de saúde pública mundial, foi observado o aumento progressivo de sua prevalência em diversos países e de acordo com o INCA ${ }^{2}$, obteve predominância de taxas nas regiões mais desenvolvidas: Sul e Sudeste. No entanto, o Amapá se equipara ao cenário nacional corroborando com os dados brasileiros, se colocando entre os assuntos de maior debate na Atenção Primária ${ }^{16}$.

Em 2013, foi a segunda causa de mortalidade por doença oncológica no sexo masculino com 14,1 óbitos por cem mil homens, atrás apenas do câncer de traqueia, brônquios e/ou pulmões, com 16,12 óbitos a cada cem mil homens ${ }^{8}$.

Para explicar o aumento nas taxas incidentes de câncer de próstata no Brasil, os principais fatores, foram: aumento da expectativa de vida da população, melhoria na qualidade dos registros, maior disponibilidade de métodos diagnósticos e aumento do diagnóstico da doença em razão da disseminação do rastreamento com teste do Antígeno Prostático Específico (PSA) e toque retal ${ }^{2}$

Neste estudo, (5,3\%) dos prontuários estudados referendavam dados de recidiva, um acontecimento possível a 
pessoa com doença oncológica. Caire et al. ${ }^{17}$ relatam que o prolongamento do tratamento causada pela recidiva, gera déficit de autoestima com profundos traços de ansiedade.

Destarte, é importante acompanhar o número e a evolução dos casos tratados em uma determinada localidade para melhor caracterização do perfil das pessoas em seguimento, tendo em vista que o estudo em questão identificou (75\%) dos casos categorizados como em seguimento, (16\%) em terapêutica paliativa e (9\%) não realizaram nenhuma terapêutica. No que se refere ao tratamento há outra demanda crucial, a UNACON não conta ainda com radioterapia, modalidade comum utilizada em associação terapêutica, sendo necessário o deslocamento da pessoa com doença oncológica a outros estados.

Os cuidados de enfermagem no seguimento oncológico estão voltados para a prevenção e controle, a realização de cuidados paliativos, o tratamento, a reabilitação e atendimento aos familiares, atuando também no desenvolvimento de ações educativas, administrativas e gerenciais. Averiguando os principais cuidados de enfermagem, existem procedimentos com (100\%) de ocorrência, como tarefas administrativas e gerenciais do cuidado, mas, também procedimentos exclusivos ao enfermeiro, como as sondagens, com (8\%) de ocorrência. Estudo realizado com 29 participantes em cuidados paliativos, corrobora com o amplo conjunto de intervenções de enfermagem, principalmente, no cuidado aos indivíduos e seus familiares e na gestão ${ }^{18}$.

\section{Limitações do estudo}

Este estudo apresenta como limitação a falta de algumas informações nos prontuários, principalmente nas anotações de enfermagem, impossibilitando uma análise mais eficaz quanto os cuidados prestados.

\section{Contribuições para a prática}

A contribuição do estudo para a área de enfermagem advém da identificação do perfil epidemiológico, sociodemográfico e clínico e os cuidados de enfermagem cujo conhecimento subsidiará o planejamento de estratégias preventivas, a fim de minimizar a incidência de cânceres, bem como elaborar a sistematização da assistência de enfemagem que estão relacionadas com as peculiaridades locais. Isso pode favorecer possiveis politicas públicas locais que visem aperfeiçoar os programas de prevenção, diagnóstico e tratamento precoce dos câanceres mais prevalentes no Estado do Amapá.

\section{CONCLUSÃO}

A partir dos resultados concluídos, foram identificadas as características epidemiológicas, clínicas e os cuidados de enfermagem nas pessoas acometidas por câncer atendidas na UNACON. O perfil socidemográfico em sua maioria se assemelha ao perfil descrito na literatura, estando o sexo feminino como o mais acometido por doença oncológica, na faixa etária entre 60-65 anos de idade, os que têm companheiros (as), a escolaridade baixa constatado pelo maior percentual de pessoas que cursaram até o ensino fundamental, situação trabalhista sem ocupação fixa, e a procedência maior indice da capital Macapá. No perfil clínico foi identificada para as categorias situação a maioria estava em seguimento, sobre o tipo de tratamento outras terapêuticas ocupam destaque, o tempo entre o diagnóstico e tempo de inicio do tratamento prevaleceu menor que um ano.

Para os tipos de cânceres, para sexo feminino predominou o câncer de colo uterino, e para o sexo masculino o câncer de próstata. Foi constatado que monitorização e controles foram os cuidados mais evidenciados na descrição nos prontuários analisados. Esses resultados podem contribuir para o planejamento de estratégias para medidas preventivas, terapêuticas e outras ações para melhor assistência, visto que se identificou particularidades comparadas ao âmbito nacional. Há necessidade de mais estudos sobre a temática, principalmente à nível estadual, pois os dados locais mostram situações específicas.

Observou-se a incidência de cânceres que estão diretamente relacionados à falta de saneamento básico, baixo nivel de escolaridade, maus hábitos alimentares e falhas no sistema de saúde, sendo estes, fatores presentes na Região Norte. Com ênfase, no câncer de estômago, que apresenta elevada ocorrência na população do estado do Amapá, justificado pelos fatores pertinentes à localidade. Contudo, ressalta-se a necessidade de programas de prevenção, diagnóstico e tratamento precoce, com ações de saúde que envolvam a equipe multidisciplinar e os órgãos públicos, para que se obtenha educação continuada e planejamento de assistência eficaz.

Contribuições dos Autores: Walter de Souza Tavares, Lucyelle Gleyce Ferreira Pádua e Mayrla Santana Picanço foram responsáveis pela concepção e/ou desenho do estudo, coleta, análise e interpretação dos dados, redação e/ou revisão crítica do manuscrito e aprovação da versão final a ser publicada. Francineide Pereira da Silva Pena e Maria Virginia Filgueiras de Assis Melo foram responsáveis pela redação e/ou revisão crítica do manuscrito e aprovação da versão final a ser publicada. 


\section{REFERÊNCIAS}

1. Organização Mundial da Saúde (OMS). Câncer mata 8,8 milhões de pessoas anualmente no mundo [Internet]. 2017 [acesso em 2018 fev 2]. Disponivel em: https://bit.ly/2kJXWy8.

2. Instituto Nacional de Câncer José Alencar Gomes da Silva (BR). Estimativa 2018: incidência de câncer no Brasil. [Internet]. Rio de Janeiro: INCA; 2017 [acesso em 2018 fev 8]. Disponível em: https://bit.ly/2Gf6Z6I. 3. Guerra MR, Bustamante-Teixeira MT, Corrêa CSL, Abreu DMX de, Curado MP, Mooney M, et al. Magnitude e variação da carga da mortalidade por câncer no Brasil e Unidades da Federação, 1990 e 2015 . Rev Bras Epidemiol. [Internet]. 2017 [acesso em 2018 jun 4]; 20(1):102-15. Disponivel em: https://bit.ly/2EiqZ5J.

4. Oliveira MM, Malta DC, Guauche H, Moura L, Silva GA. Estimativa de pessoas com diagnóstico de câncer no Brasil: dados da Pesquisa Nacional de Saúde, 2013. Rev Bras Epidemiol [Internet]. 2015 [acesso em: 2018 dez 6]; 18(2):146-57. Disponivel em: https://bit.ly/2rBTJly.

5. Silva DVA, Carmo JR, Cruz MÉA, Rodrigues CAO, Santana ET, Araújo DD. Caracterização clínica e epidemiológica de pacientes atendidos por um programa público de atenção domiciliar. Enferm. Foco [Internet]. 2019 [acesso em 2020 jun 08]; 10 (3): 112-118. Disponivel em: https://doi.org/10.21675/2357707X.2019.v10.n3

6. Carvalho MHR, Carvalho SMR, Laurenti R, Payão SLM. Tendência de mortalidade de idosos por doenças crônicas no município de Marília-SP, Brasil: 1998 a 2000 e 2005 a 2007. Epidemiol Serv Saúde [Internet] 2014 [acesso em: 2018 dez 6];23(2):347-54. Disponivel em: https://bit.ly/2BfODhO.

7. Cardoso AC, Noguez PT, SG Oliveira, Porto AR, Perboni JS, Farias TA. Rede de apoio e sustentação dos cuidadores familiares de pacientes em cuidados paliativos no domicílio. Enferm. Foco [Internet]. 2019 [acesso em 2020 jun 08]; 10 (3): 34-39. Disponivel em: https://doi.org/10.21675/2357-707X.2019.v10.n3.

8. Andrade FP, Muniz RM, Lange C, Guanilo MEE. Perfil sociodemográfico e econômico dos sobreviventes ao câncer segundo o grau de resiliência. Texto \& contexto enferm [Internet]. 2013 [acesso em 2018 dez 6];22(2):476-84. Disponivel em: https://bit.ly/2RWrzKL.

9. Figueiredo T, Silva AP, Silva RMR, Jesus Silva J, Oliveira CS, Alcântara DDF, Souza AAM, et al. Como posso ajudar? Sentimentos e experiências do familiar cuidador de pacientes oncológicos. ABCS Health Sci [Internet]. 2017 [acesso em 2018 dez 10];42(1):34-9. Disponivel em: https://bit.ly/2EhO6FO.

10. Batiston AP, Tamaki EM, Souza LA, Santos MLM. Conhecimento e prática sobre os fatores de risco para o câncer de mama entre mulheres de 40 a 69 anos. Rev Bras Saúde Mater Infant [Internet]. 2011 [acesso em 2018 dez 6];11(2):163-71. Disponivel em: https://bit.ly/2SNHDOL.

11. Malta DC, Bernal RTI. Comparação dos fatores de risco e proteção de doenças crônicas na população com e sem planos de saúde nas capitais brasileiras, 2011. Rev Bras Epidemiol. [Internet]. 2014 [acesso em 2018 dez 6]; 17(1):241-55. Disponível em: https://bit.ly/2Gf7gXl.

12. Ferlay J, Parkin DM, Pisani P. GLOBOCAN 2012: Cancer incidence and mortality worldwide. Lyon: IARC [Internet]. 2013 [acesso em $2018 \mathrm{dez}$ 6]. Disponivel em: http://globocan.iarc.fr.

13. Barbosa IR, Souza DLB, Bernal MM, Costa ICC. Desigualdades regionais na mortalidade por câncer de colo de útero no Brasil: tendências e projeções até o ano 2030. Ciênc Saúde Coletiva [Internet]. 2016 [acesso em 2018 dez 6];21(1):253-62. Disponivel em: https://bit.ly/2Gg3PiT.

14. Sales ML, Guimarães AVS. Fatores de risco no desenvolvimento de câncer gástrico: uma análise na população brasileira. Rev Bras Ciênc Vida [Internet]. 2017 [acesso em 2018 dez 6];5(1). Disponível em: https://bit.ly/2zU5KUQ.

15. Mendes AAR, dos Santos FCT, Assis MO, Monteiro R, Garcia Junior SS, Neves WS, da Silva MHS, et al. Avaliação da sobrevida de pacientes com câncer do trato gastrointestinal em uma cidade do interior de Minas Gerais. Rev Med Minas Gerais [Internet]. 2018 [acesso em 2018 Dez 6];28(4):5-11. Disponivel em: http://www.rmmg.org/artigo/detalhes/2301.

16. Bidinotto DNPB. A saúde do homem: doenças crônicas não transmissíveis e vulnerabilidade social. Rev Latinoam Enferm [Internet]. 2015 [acesso em 2018 dez 6];24:e2756. Disponível em: http://www.scielo.br/ pdf/rlae/v24/pt_0104-1169-rlae-24-02756.pdf.

17. Caires S, Machado M, Antunes MC, Melo ASM. Recidiva Oncológica: Olhares dos Profissionais Hospitalares sobre as Dificuldades do Paciente Pediátrico. Psico-USF [Internet]. 2018 [acesso em 2018 dez 10];23(2):333-45. Disponível em: https://bit.ly/2zSUrfy.

18. Fuly PDSC, Pires LMV, de Souza COS, Renaud BG, de Oliveira B, Padilha, KG. Carga de trabalho de enfermagem de pacientes oncológicos sob cuidados paliativos. Rev Esc Enf USP [Internet]. 2016 [acesso em 2018 maio 18];50(5):793-800. Disponivel em: https://bit.ly/2BeYXow. 